\title{
KECK OBSERVATIONS OF THE GALACTIC CENTER SOURCE G2: GAS CLOUD OR STAR?
}

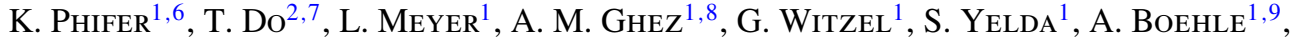 \\ J. R. Lu ${ }^{3,10}$, M. R. Morris ${ }^{1}$, E. E. BeCKLIN ${ }^{1,4}$, AND K. MatTHEws ${ }^{5}$ \\ ${ }^{1}$ Department of Physics and Astronomy, University of California, Los Angeles, CA 90095, USA; ghez@astro.ucla.edu \\ ${ }^{2}$ Dunlap Institute for Astronomy and Astrophysics, University of Toronto, Toronto, Ontario, Canada M5S 3H4 \\ ${ }^{3}$ Institute for Astronomy, University of Hawaii, Hilo, HI 96720, USA \\ ${ }^{4}$ NASA-Ames Research Center, Mountain View, CA 94035, USA \\ ${ }^{5}$ Division of Physics, Mathematics, and Astronomy, California Institute of Technology, Pasadena, CA 91125, USA \\ Received 2013 April 17; accepted 2013 July 7; published 2013 July 29
}

\begin{abstract}
We present new observations and analysis of G2-the intriguing red emission-line object which is quickly approaching the Galaxy's central black hole. The observations were obtained with the laser guide star adaptive optics systems on the W. M. Keck I and II telescopes (2006-2012) and include spectroscopy $(R \sim 3600)$ centered on the hydrogen $\mathrm{Br} \gamma$ line as well as $K^{\prime}(2.1 \mu \mathrm{m})$ and $L^{\prime}(3.8 \mu \mathrm{m})$ imaging. Analysis of these observations shows the $\mathrm{Br} \gamma$ line emission has a positional offset from the $L^{\prime}$ continuum. This offset is likely due to background source confusion at $L^{\prime}$. We therefore present the first orbital solution derived from $\mathrm{Br} \gamma$ line astrometry, which, when coupled with radial velocity measurements, results in a later time of closest approach (2014.21 \pm 0.14$)$, closer periastron (130 AU, $1600 R_{\mathrm{s}}$ ), and higher eccentricity $(0.9814 \pm 0.0060)$ compared to a solution using $L^{\prime}$ astrometry. It is shown that G2 has no $K^{\prime}$ counterpart down to $K^{\prime} \sim 20$ mag. G2's $L^{\prime}$ continuum and the Br $\gamma$ line emission appears unresolved in almost all epochs, which implies that the bulk of the emission resides in a compact region. The observations altogether suggest that while G2 has a gaseous component that is tidally interacting with the central black hole, there is likely a central star providing the self-gravity necessary to sustain the compact nature of this object.
\end{abstract}

Key words: accretion, accretion disks - black hole physics - Galaxy: center - Galaxy: kinematics and dynamics infrared: general

\section{INTRODUCTION}

Recently, Gillessen et al. $(2012,2013)$ reported the discovery of G2, an extremely red object with spatially resolved $\mathrm{Br} \gamma$ emission. The object was interpreted as a $\sim 3$ Earth-mass gas cloud based upon an inferred low dust temperature, observed elongation of the $\mathrm{Br} \gamma$ emission along the object's direction of motion, and a claimed tail along the same orbital trajectory as G2. This interpretation is particularly interesting because G2 is on a highly eccentric orbit with a closest approach to our Galaxy's central black hole within the next year, potentially allowing us to observe an unprecedented accretion event onto a supermassive black hole and offer insight into the region surrounding the black hole (e.g., Morris 2012; Mościbrodzka et al. 2012; Anninos et al. 2012; Saitoh et al. 2012; Bartos et al. 2013; Yusef-Zadeh \& Wardle 2013).

The interpretation of G2 as a gas cloud, however, is not definitive. One challenge for the pure gas cloud scenario is that, given the strong tidal fields in this region and G2's low selfgravity, G2 must have formed quite recently $(\sim 1995$, just prior to the initial observations; Burkert et al. 2012; Schartmann et al. 2012). Since such a gas cloud would be tidally disrupted during its periapse passage in the upcoming year, the gas cloud model implies that $\mathrm{G} 2$ will be observed over almost the entire extent of its existence. Therefore, several alternative scenarios invoking an underlying star have been proposed (Miralda-Escudé 2012;

\footnotetext{
6 NSF Graduate Student Fellow, Grant DGE-0707424.

7 Dunlap Fellow.

8 Leichtman-Levine Chair in Astrophysics.

9 Preston Fellow.

${ }^{10}$ NSF Astronomy and Astrophysics Postdoctoral Fellow, Grant AST-1102791.
}

Murray-Clay \& Loeb 2012; Scoville \& Burkert 2013). In these scenarios, the observed heated gas is posited to be circumstellar, either intrinsic or a consequence of interaction of the star and surrounding ambient gas. In these stellar scenarios, G2 not only has existed for timescales much longer than the observed time baseline, but the continuum source $\left(L^{\prime}\right)$ will also survive periapse passage.

Regardless of its nature, G2's properties and possible origin depend critically on its orbital parameters. These parameters have been estimated from observations with a short time baseline compared to the orbital period ( $\sim 10$ versus $\sim 200 \mathrm{yr}$; Gillessen et al. 2013) and in a very crowded region, making the orbital solution susceptible to biases (Hartkopf et al. 2001). We therefore present new measurements and analysis of G2 that minimize the effects of source confusion on estimates of G2's orbital parameters and examine the temporal evolution of G2's properties.

\section{OBSERVATIONS}

Two types of new data were collected for this study using the laser guide star adaptive optics (LGS AO) systems at the W.M. Keck Observatory (Wizinowich et al. 2006; van Dam et al. 2006; Chin et al. 2012). Spectra were obtained using the OSIRIS integral field spectrograph (Larkin et al. 2006) through the narrowband $\mathrm{Kn} 3$ filter, which is centered on the $\mathrm{Br} \gamma$ hydrogen line $(2.1661 \mu \mathrm{m})$, at a spectral resolution of $R \sim 3600$. Imaging data were obtained in the $K^{\prime}$-band filter $\left(\lambda_{0}=2.124 \mu \mathrm{m}\right)$ and the $L^{\prime}$-band filter $\left(\lambda_{0}=3.776 \mu \mathrm{m}\right)$ using the Keck II nearinfrared camera (NIRC2; PI: K. Matthews). These data were obtained and reduced in a similar manner as in our previous publications (Ghez et al. 2005b; Hornstein et al. 2007; Ghez et al. 2008; Do et al. 2009; Lu et al. 2009; Yelda et al. 2010; 
Table 1

Summary of Observations and Measurements of G2

\begin{tabular}{|c|c|c|c|c|c|c|c|c|c|c|c|}
\hline UT Date & $\begin{array}{c}\text { Fractional } \\
\text { Date }\end{array}$ & $\begin{array}{l}\text { AO Type/ } \\
\text { Telescope }\end{array}$ & $\begin{array}{c}\text { Pixel Scale } \\
\text { (mas) }\end{array}$ & $\begin{array}{c}N_{\text {frames }} \\
\text { Observed }\end{array}$ & $\begin{array}{c}N_{\text {frames }} \\
\text { Used }\end{array}$ & $\begin{array}{c}\text { FWHM } \\
\text { (mas) }\end{array}$ & $\begin{array}{l}\text { Orig } \\
\text { Pub. }^{\text {a }}\end{array}$ & $\begin{array}{c}\text { vlsr } \\
\left(\mathrm{km} \mathrm{s}^{-1}\right)\end{array}$ & $\begin{array}{l}\mathrm{Br} \gamma \mathrm{FWHM} \\
\quad\left(\mathrm{km} \mathrm{s}^{-1}\right)\end{array}$ & $\begin{array}{c}\mathrm{G} 2 \Delta \mathrm{R} . \mathrm{A} \cdot{ }^{\mathrm{b}} \\
\text { (mas) }\end{array}$ & $\begin{array}{c}\text { G2 } \Delta \text { Decl. } \\
\text { (mas) }\end{array}$ \\
\hline \multicolumn{12}{|c|}{ OSIRIS, Kn3 } \\
\hline 2006 Jun 18, 30; Jul 1 & 2006.495 & Keck II LGS & 35 & 28 & 27 & 74 & 3 & $1125 \pm 6$ & $137 \pm 16$ & $222.97 \pm 5.05$ & $-105.40 \pm 2.40$ \\
\hline 2008 May 16; Jul 25 & 2008.487 & Keck II LGS & 35 & 22 & 21 & 78 & 4,0 & e & $\mathrm{e}$ & $181.82 \pm 6.90$ & $-64.97 \pm 2.51$ \\
\hline 2009 May 5, 6 & 2009.344 & Keck II LGS & 35 & 24 & 19 & 79 & 0 & $1352 \pm 20$ & $163 \pm 28$ & $176.26 \pm 2.74$ & $-65.76 \pm 2.04$ \\
\hline 2010 May 5, 8 & 2010.349 & Keck II LGS & 35 & 17 & 16 & 82 & 0 & $1479 \pm 18$ & $256 \pm 33$ & $166.29 \pm 9.11$ & $-43.95 \pm 11.05$ \\
\hline 2012 Jun 9, 11; Aug 11, 12 & 2012.613 & Keck I LGS & $20^{\mathrm{c}}$ & 27 & $21^{d}$ & 68 & 0 & $2071 \pm 86$ & $726 \pm 111$ & $103.16 \pm 4.07$ & $-8.79 \pm 11.36$ \\
\hline \multicolumn{12}{|c|}{ NIRC2, $L^{\prime}$} \\
\hline 2003 Jun 10 & 2003.440 & Keck II NGS & 10 & 12 & 12 & 86 & 0 & & & $260.1 \pm 4.5$ & $-154.6 \pm 5.1$ \\
\hline 2004 Jul 26 & 2004.567 & Keck II LGS & 10 & 11 & 11 & 80 & 1 & & & $262.6 \pm 4.8$ & $-140.4 \pm 4.2$ \\
\hline 2005 Jul 30 & 2005.580 & Keck II LGS & 10 & 62 & 56 & 87 & 2 & & & $251.6 \pm 3.4$ & $-129.0 \pm 2.6$ \\
\hline 2006 May 21 & 2006.385 & Keck II LGS & 10 & 19 & 19 & 81 & 0 & & & $226.2 \pm 1.9$ & $-99.4 \pm 0.8$ \\
\hline 2009 Jul 22 & 2009.561 & Keck II LGS & 10 & 4 & 4 & 85 & 0 & & & $175.4 \pm 4.9$ & $-70.7 \pm 2.1$ \\
\hline 2012 Jul 20-23 & 2012.562 & Keck II LGS & 10 & 1316 & 1314 & 93 & 0 & & & $105.9 \pm 0.8$ & $-21.1 \pm 0.6$ \\
\hline
\end{tabular}

Notes.

a References: (0) this Work; (1) Ghez et al. (2005a); (2) Hornstein et al. (2007); (3) Ghez et al. (2008); (4) Do et al. (2009).

b Offset from SgrA*-radio.

c A smaller square dither pattern of $0.5 \times 0.5$ was used.

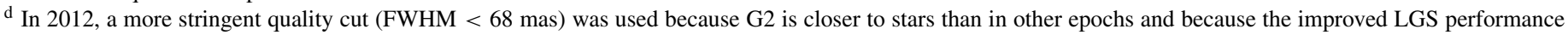
on Keck I allowed this more stringent cut.

e In 2008, low signal-to-noise ratio and changes in the local standard of rest velocity between the two observation dates prevent reliable line measurements.

Meyer et al. 2012) and the specific spectroscopic and $L^{\prime}$ observations utilized in this Letter are described in Table 1.

\section{ANALYSIS}

\subsection{OSIRIS IFU Measurements}

Because $\mathrm{G} 2$ has no detectable $K$ continuum and is fainter than any object we have previously extracted, some analysis steps differ from our earlier analyses. For all epochs, we created a combined data cube before extracting G2's spectrum rather than extracting spectra from individual cubes. The $\mathrm{OH}$ sky lines in the data are subtracted using sky frames scaled to the strength of families of $\mathrm{OH}$ lines in the observed frames to account for temporal variations. In 2012, the $\mathrm{Br} \gamma$ emission line from G2 is coincident with a prominent $\mathrm{OH}$ sky line at $2.180 \mu \mathrm{m}$, so to minimize the systematic effects associated with $\mathrm{OH}$ line subtraction, we scale the sky only to this line.

An iterative process was required to estimate the position and spectral properties of G2 in the OSIRIS data cubes (see Figures 1 and 2) since G2's position is needed to place the aperture for spectral extraction, and G2's spectral properties are needed to determine which OSIRIS channels should be used to measure its position. G2's position was first obtained by visual inspection of the data cube. Then, an initial spectrum was extracted at this position using an aperture with a radius of 35 mas. Emission from local ambient gas was subtracted using a region free of stellar halos within $\sim 0$ '.5. A Gaussian was fit to the resulting emission line. In order to refine the position, the three-dimensional data cube was median collapsed over the wavelength range corresponding to twice the standard deviation of the Gaussian fit to the emission line, centered on the line peak. Continuum emission was subtracted by averaging the median of the 25 nearest channels on either side of the line. G2's position was further refined with a two-dimensional Gaussian fit to the continuum-subtracted image. The iterative extraction process was repeated again to obtain a final position

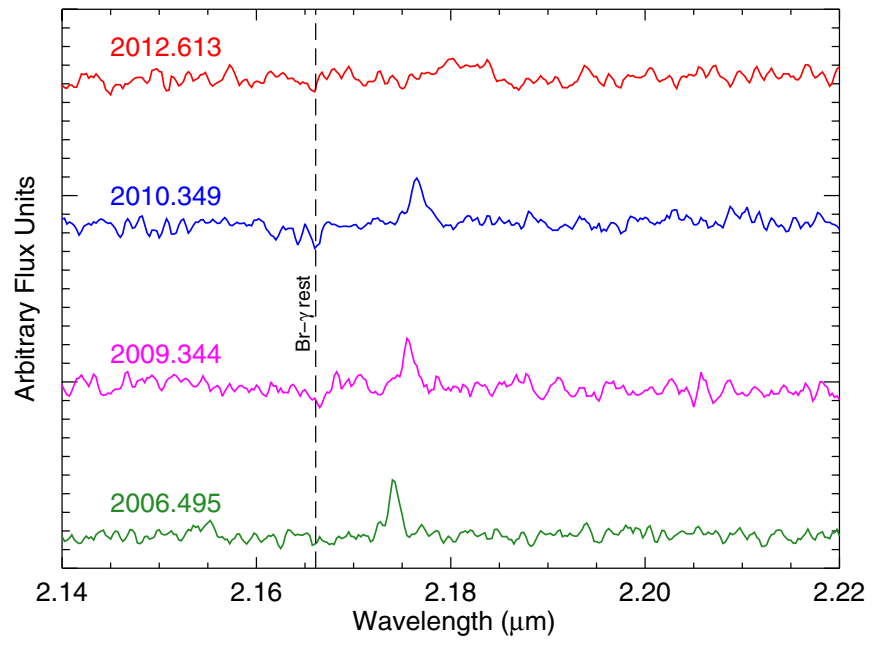

Figure 1. The evolving spectra of G2. The highly redshifted $\mathrm{Br} \gamma$ emission line has a FWHM that increases with time (see Table 1). The Br $\gamma$ line peak has been scaled such that the flux scales are constant. Additionally, the spectra have been smoothed using a boxcar average with a width of three OSIRIS channels $\left(7.5 \times 10^{-4} \mu \mathrm{m}\right)$.

and spectrum for G2. Further iterations produced no significant changes. Measuring G2's position on a continuum-subtracted frame (see Figure 2(b)) removes the effect of source confusion, thereby avoiding astrometric biases.

G2's spectral properties were obtained using the final Gaussian fits to the $\mathrm{Br} \gamma$ emission line. The radial velocities (RVs) for G2 were calculated from the offset of the line from the rest wavelength $\left(\lambda_{\mathrm{Br} \gamma}=2.1661 \mu \mathrm{m}\right)$ and corrected to the local standard of rest. The reported FWHM measurements were corrected for instrumental broadening $\left(\mathrm{FWHM}=85 \mathrm{~km} \mathrm{~s}^{-1}\right)$. Line flux measurements were made by comparing the integral of the Gaussian fit to the integral of the flux density of the non-variable star S0-2 ( $F_{\text {observed, } K^{\prime}}=1.46 \pm 0.02 \mathrm{mJy}$; Ghez et al. 2008) over the wavelength range of $2.17-2.18 \mu \mathrm{m}$. Dereddened fluxes were 


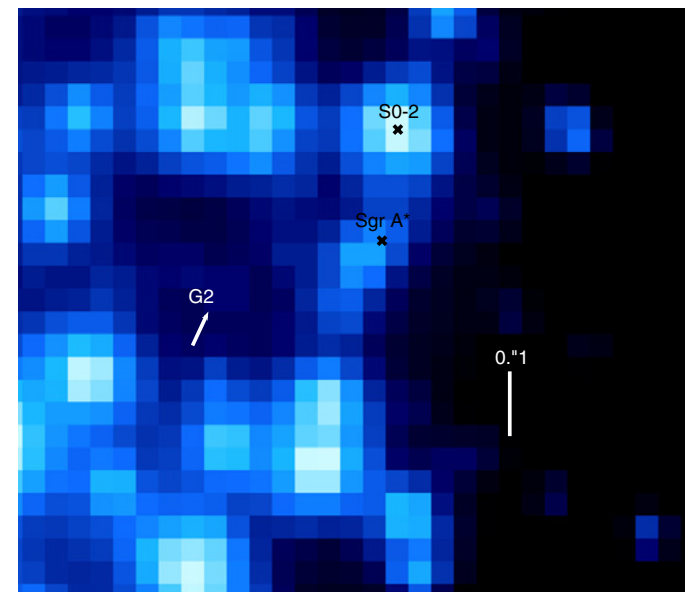

(a)

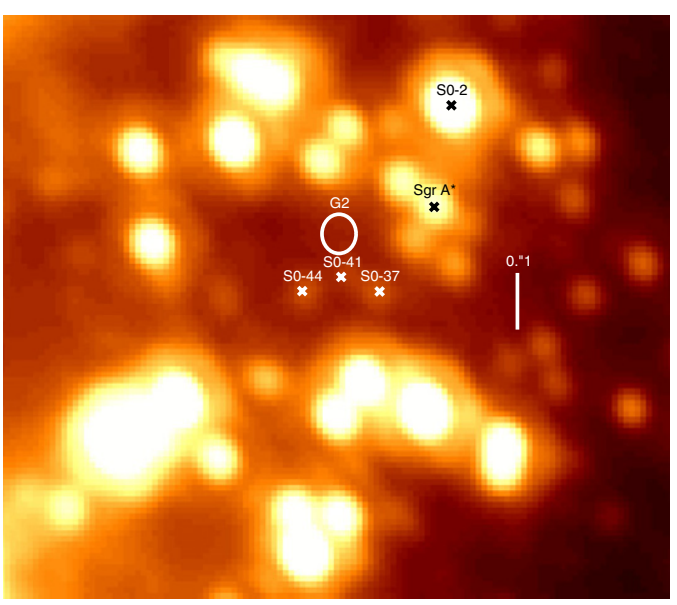

(c)

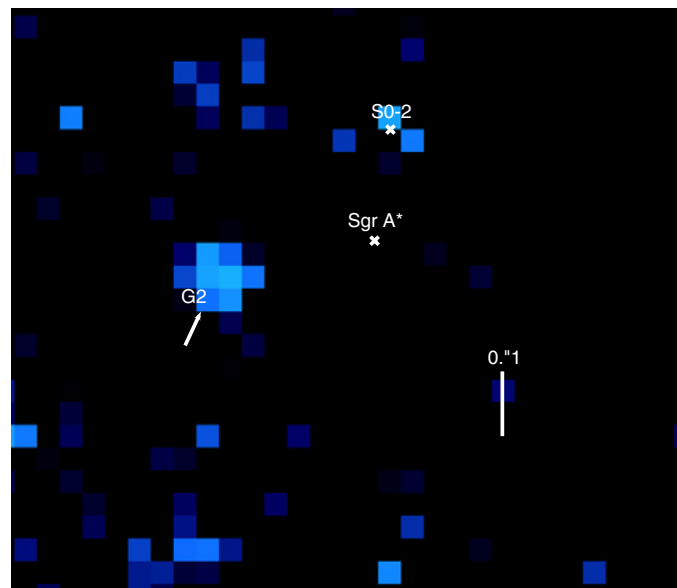

(b)

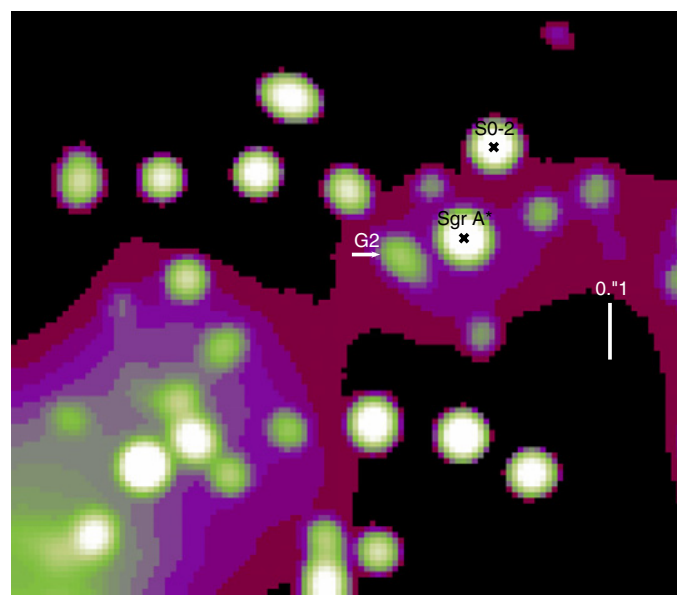

(d)

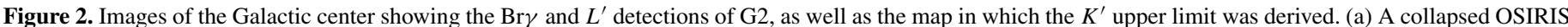

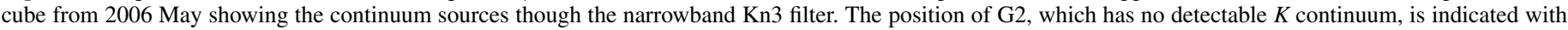

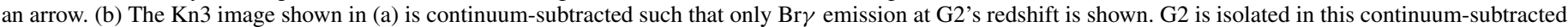

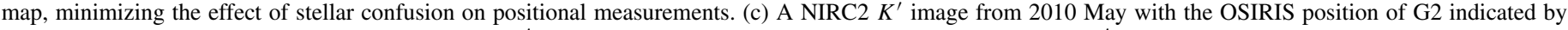

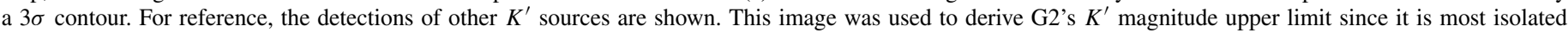

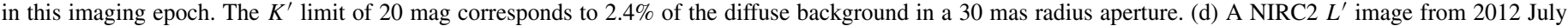

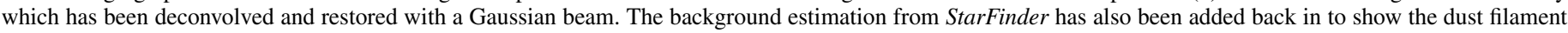

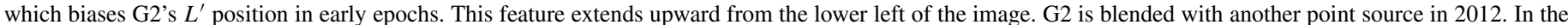
non-deconvolved images, G2 is three to four times higher than the extended background ( $\left.r_{a} p=30 \mathrm{mas}\right)$.

computed assuming an extinction value of $A_{K^{\prime}}=2.46 \pm 0.03$ (Schödel et al. 2010). FWHM and line flux uncertainties are based on the formal errors from the Gaussian fits and the RV uncertainties are from the error on the mean from the three subsets.

The final $\mathrm{Br} \gamma$ line maps were used to evaluate G2's physical size and absolute astrometric position. The size of G2's Br $\gamma$ emission was estimated by comparing the FWHM of G2 to that of nearby stars. To track the motions of G2 in the plane of the sky, its OSIRIS position was transformed into an absolute coordinate system. To construct an absolute reference frame, we used the precise locations of well-characterized stars in the field (see Figure 2(a)), as measured with StarFinder (Diolaiti et al. 2000). The position of G2 from the two-dimensional Gaussian fit was added to the list of positions for each epoch. Then, these positions were matched to those from LGS AO $K^{\prime}$ observations of the same region at a nearby epoch using a second-order polynomial transformation to account for translation, rotation, and pixel-scale differences between the images. ${ }^{11}$ Once the positions in the OSIRIS frames were transformed into a $K^{\prime}$ epoch, they were transformed into an absolute reference frame in which SgrA*-radio is at rest at the origin, as originally described in Yelda et al. (2010) and updated in Yelda et al. (2013). We investigated possible systematic astrometric effects in OSIRIS by cross-checking the OSIRIS astrometry of S0-2 to our standard NIRC2 analysis. The results are on average consistent at the $1 \sigma$ level, validating the use of OSIRIS astrometry.

\subsection{NIRC2 Imaging Measurements}

The $L^{\prime}$ position of G2 was estimated from calibrated nearinfrared images in a similar fashion to that described above for the $\mathrm{Br} \gamma$ line maps. One difference was that we deconvolved the individual background-subtracted images using the

\footnotetext{
11 In 2012, only a linear transformation was used because fewer reference stars were available due to the smaller field of view (7 versus 19-27 in other epochs).
} 
Lucy-Richardson algorithm (Richardson 1972; Lucy 1974; Lucy \& Hook 1992) in order to help isolate the point sources from the extended $L^{\prime}$ dust emission. Both the large-scale background and the point-spread function (PSF) were estimated using StarFinder. After beam-restoring the individual frames with a Gaussian having FWHM of half the theoretical resolution limit (40 mas), the frames were averaged to create a final image for each epoch (see Figure 2(d)). The results of this analysis do not vary if the image is restored using a wider Gaussian beam. To locate stars used as astrometric reference sources, StarFinder was run on the combined (deconvolved) frame using the beamrestoring Gaussian as the reference PSF. G2's position was determined from an elliptical two-dimensional Gaussian fit. ${ }^{12}$ The fitting of an elliptical Gaussian allows for extended structure and is analogous to the method used for the $\mathrm{Br} \gamma$ detection. The positions for $\mathrm{G} 2$ from this method are consistent with those produced via StarFinder. The size of G2 was measured by comparing to the PSF of stars with a similar flux. The observed $L^{\prime}$ positions were transformed into an absolute coordinate system as described for the $\mathrm{Br} \gamma$ detections. The $L^{\prime}$ fluxes were calibrated as described in Witzel et al. (2012).

One complication for measuring the $L^{\prime}$ position of $\mathrm{G} 2$ in the plane of the sky is that G2 was superimposed on a filament of dust emission during the early to mid-2000s, which caused significant astrometric bias (see Figure 2(d)). To quantify this effect, we performed a series of star-planting simulations using a 2012 May $L^{\prime}$ image, in which G2 is well off the filament. In each of the four simulations, an extra point source $\left(L^{\prime}=14\right)$ was planted at the expected G2 position at the time of earlier $L^{\prime}$ observations (2003.440, 2004.567, 2005.580, and 2006.385 ) based upon the orbit fit to the $\mathrm{Br} \gamma$ measurements. The background dust emission does not have noticeable motions in the plane of the sky, thus the astrometric bias at these locations in the 2012 image should be representative of the bias at earlier epochs. The position of the artificial point source was extracted using StarFinder.

In the NIRC2 $K^{\prime}$ images, no $K^{\prime}$ counterpart is detected for G2 and star-planting simulations were carried out to determine an $K^{\prime}$ upper limit. We used the $\mathrm{Br} \gamma$ astrometry of $\mathrm{G} 2$ to predict the positions of $\mathrm{G} 2$ in the $K^{\prime}$ data. Then, an artificial point source was planted at G2's predicted position in the 2010 May image, when G2 is most isolated (see Figure 2(c)). Using a modified version of the StarFinder algorithm (A. Boehle et al., in preparation), we searched for a point source within a 3 pixel box centered on the planted star, given the PSF originally extracted from the 2010 May image and using a $3 \sigma$ detection threshold.

\subsection{Orbital Modeling}

We fit an orbit to G2's astrometric positions and RV measurements. $L^{\prime}$-astrometric and RV measurements reported by Gillessen et al. $(2012,2013)$ were also included ${ }^{13}$; however, we fit the $\mathrm{Br} \gamma$ and $L^{\prime}$ astrometry separately. We assumed G2 follows a purely Keplerian orbit around the central black hole. Since the observed motion of G2 does not entail enough information to independently well constrain the properties of the black hole (mass, distance along the line- of sight, position in the plane of the sky, and three-dimensional velocity), the motion of

\footnotetext{
12 In 2012, a two-component elliptical Gaussian was fit since G2 is blended with another point source (separation $\sim 40$ mas); the component assigned to $\mathrm{G} 2$ was based on the positional proximity to the position predicted by the $\mathrm{Br} \gamma$ orbit.

13 These data are publicly available:

https://wiki.mpe.mpg.de/gascloud/PlotsNData.
}

Table 2

Orbital Elements for $\mathrm{G}^{\mathrm{a}}$

\begin{tabular}{lc}
\hline \hline Parameter & Value $^{\mathrm{b}}$ \\
\hline Time of closest approach $\left(T_{0}\right)$ & $2014.21 \pm 0.14 \mathrm{yr}$ \\
Eccentricity $(e)$ & $0.9814 \pm 0.0060$ \\
Period $(P)$ & $276 \pm 111 \mathrm{yr}$ \\
Angle to periapse $(\omega)$ & $88^{\circ} \pm 6^{\circ}$ \\
Inclination $(i)$ & $121^{\circ} \pm 3^{\circ}$ \\
Position angle of the ascending node $(\Omega)$ & $56^{\circ} \pm 11^{\circ}$ \\
\hline
\end{tabular}

Notes.

a Parameters describing the gravitational potential are found in Meyer et al. (2012).

b Values provided are the mean and standard deviation of the marginalized one-dimensional distributions.

S0-2, which has undergone a full orbit, was fit simultaneously and effectively determines these parameters. In this way, the remaining orbital parameters for G2 (eccentricity, period, time of periapse passage, inclination, position angle of the ascending node, and the longitude of periapse) are well determined from the data (see, e.g., Ghez et al. 2005b; Gillessen et al. 2012; Meyer et al. 2012). Compared to earlier publications, we amended our Keplerian orbit fitting code to use the Bayesian sampler MultiNest (Feroz \& Hobson 2008); we verified the results are equivalent with our previous Monte Carlo approach (e.g., Ghez et al. 2008).

\section{RESULTS}

Six key results emerge from our analysis of $\mathrm{G} 2$.

1. Apparent positional offset between the Bry line emission and $L^{\prime}$ continuum emission of $G 2$. The $L^{\prime}$ astrometry is not consistent with our $\mathrm{Br} \gamma$ astrometry, which is reported here for the first time (see Figure 3(a)). The positional difference can be explained by astrometric bias in the $L^{\prime}$ astrometry. In our star-planting analysis, we find the extracted position of an artificial $L^{\prime}$ source on the dust filament where G2 is located at early epochs differs from the input positions by an average of $\sim 1.2$ pixel (12 mas). This is almost an order of magnitude larger than typical centroiding uncertainties at $L^{\prime}$ and the astrometric bias found in a region isolated from extended background flux. We therefore exclude $L^{\prime}$ astrometry from our primary orbital fits.

2. Revised orbital parameters. The Keplerian orbit derived using $\mathrm{Br} \gamma$ astrometry differs from the orbit derived using $L^{\prime}$ astrometry (e.g., Gillessen et al. 2013) by more than can be explained by formal measurement uncertainties (see Figure 3(a)). We list the most likely orbital parameters from $\mathrm{Br} \gamma$ in Table 2. This new orbit for G2 has pushed the closest approach date to 2014 March (2014.21 \pm 0.14$)$, has a closer periastron (130 $\left.\mathrm{AU}, 1600 R_{\mathrm{S}}\right)$ and a higher eccentricity $(0.9814 \pm 0.0060)$. This is consistent with an $\mathrm{RV}$ only fit $\left(T_{o}=2014.47 \pm 0.46\right.$ and $\left.e=0.978 \pm 0.013\right)$.

3. G2's compact size. G2's observed size is comparable to the Keck angular resolution in almost all our observations; however, it exhibits marginal spatial extent in our highest quality spectroscopic data set. At this time (2006), the observed half-width at half-maximum is $20 \pm 4$ mas after accounting for the observed PSF of SO-2 (FWHM = $74 \pm 3$ mas), which is consistent with that presented in Gillessen et al. (2012).

4. Spectral evolution. We find an increasing $\mathrm{Br} \gamma$ line width for G2, with FWHM measurements ranging from 

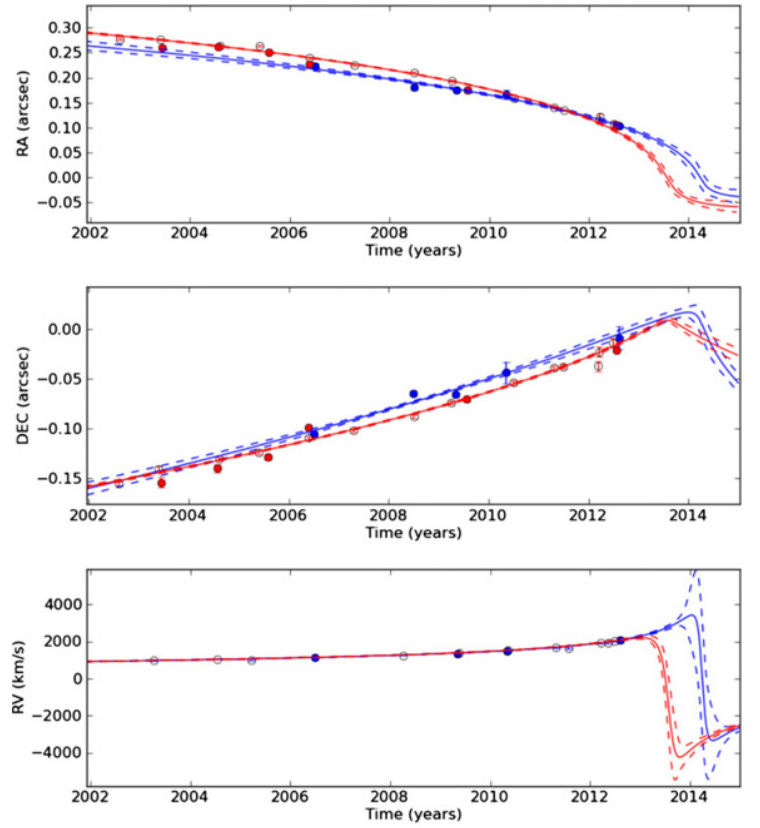

(a)

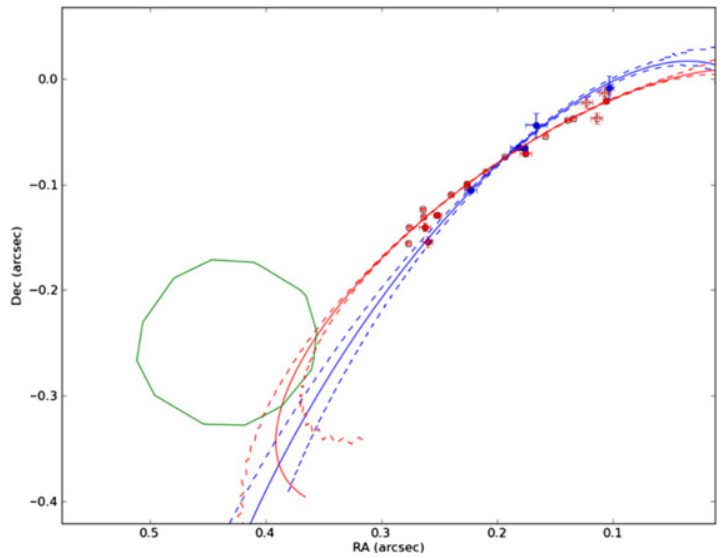

(b)

Figure 3. (a) The orbital fit using the $\mathrm{Br} \gamma$ astrometry presented in this Letter is shown in blue and the fit derived using the $L^{\prime}$ astrometry presented in Gillessen et al. $(2012,2013)$ is shown in red. Both solutions use all radial velocity fits determined from the Br $\gamma$ emission line (our+MPE). Astrometric points obtained from data presented in this Letter are indicated with filled points, and open circles indicate measurements presented in Gillessen et al. (2012, 2013). Both $L^{\prime}$ data sets appear offset from the Br $\gamma$ astrometry. (b) These two-dimensional orbits complement those shown to the left. The location of the previously claimed "tail" is indicated by a green contour. This location was computed in a continuum-subtracted frame at this object's peak velocity $\left(\sim 645 \mathrm{~km} \mathrm{~s}^{-1}\right)$ and creating a contour level which represents a flux level 10 times greater than the median pixel value in that region. As can be seen, this low surface brightness object is only marginally spatially associated with either version of G2's orbit.

$158 \pm 12 \mathrm{~km} \mathrm{~s}^{-1}$ in 2006 to $523 \pm 173 \mathrm{~km} \mathrm{~s}^{-1}$ in 2012 (see Table 1). These measurements are consistent with those presented by Gillessen et al. (2012, 2013; however, see Eckart et al. 2013).

5. Constant brightness. Both at $L^{\prime}$ and $\mathrm{Br} \gamma$ the flux of $\mathrm{G} 2$ is constant within uncertainties. The integrated flux of G2 at $L^{\prime}$ has an average value of $14.9 \pm 0.3 \mathrm{mag}$. The $\mathrm{Br} \gamma$ integrated line fluxes in 2006, 2009, 2010, and 2012 are $1.58 \pm 0.16,1.55 \pm 0.24,2.26 \pm 0.36$, and $2.17 \pm 0.32$, respectively, in units of milli- $L_{\odot}$.

6. Source color. Our star-planting simulations indicate that the upper $K^{\prime}$ magnitude limit of G2 is $K^{\prime}=20$ and is $\sim 2$ mag deeper than the earlier upper $K^{\prime}$ limit imposed on G2 (Gillessen et al. 2012). The deeper $K^{\prime}$ limit yields a dust temperature below $\sim 500 \mathrm{~K}$ if G2 is a pure gas cloud. If G2 has an underlying stellar source, a $K^{\prime}$ magnitude limit of 20 mag places distinct limits on the luminosity of the star. This limit is similar to the expected $K^{\prime}$ magnitude for a low-mass T Tauri star in the Galactic center (e.g., Scoville $\&$ Burkert 2013), and eliminates a more massive star unless it is shrouded by dust which self-extincts the star at $K^{\prime}$.

\section{DISCUSSION}

The two orbits derived by Gillessen et al. (2012, 2013) differ from each other by more than can be explained with formal measurement uncertainties. Systematic errors in the $L^{\prime}$ astrometry naturally explain the discrepancy. Because the offset becomes less significant and the RV measurements play an increasingly dominant role in the orbital modeling as G2 approaches periapse, the measurements seem to converge on the solution obtained with the less biased $\mathrm{Br} \gamma$ astrometry and $\mathrm{RV}$ measurements as well as the RV-only solution.
There are several implications of the new orbital model parameters. First, the revised periapse date of 2014 March and the closer periastron are important for the design and interpretation of $\mathrm{SgrA}^{*}$ monitoring programs designed to test for an increased accretion flow. With a special NRAO call for proposals and tremendous attention called to this object, which was originally anticipated to experience periapse in June 2013, more than 30 programs have been approved for the summer of 2013 covering radio to $\gamma$-ray wavelengths. While some models predict that a cloud would generate enhanced radio emission from SgrA* well in advance of periapse passage (e.g., Narayan et al. 2012; Sadowski et al. 2013a, 2013b), none has yet been detected (Kassim et al. 2013), although this may not yet be expected with the revised periapse passage. Thus, some monitoring in 2014 is called for.

Second, the geometry of the new orbit may call two previously suggested associations into question. Most importantly, the previous claimed "tail" of low surface brightness emission, which was substantial to the original claim that $\mathrm{G} 2$ is a pure gas cloud, is no longer securely affiliated with the compact "head," which has been analyzed in this work (see Figure 3(b)). The new orbit also falls $3.5 \sigma$ off the plane of the disk of young stars orbiting the central black hole at $\sim 0.05-2$ pc (Genzel et al. 2000; Levin \& Beloborodov 2003; Paumard et al. 2006; Lu et al. 2009; Bartko et al. 2009; Yelda et al. 2013) which has been suggested as the possible origin of the gas cloud through colliding stellar winds of young stars (Ozernoy et al. 1997; Cuadra et al. 2006) or a central stellar source (e.g., Murray-Clay \& Loeb 2012).

Third, the even higher eccentricity provides strong constraints on G2's origin. It is notable that a high eccentricity is consistent with the outcome of three-body exchanges between a binary system and the central black hole which may explain the dense concentration of B stars (the $\mathrm{S}$ stars) in the innermost regions 
of the Galaxy (e.g., Alexander \& Livio 2004). G2's observed physical properties (red, compact, and at times marginally resolved) are also consistent with the expected observables for stars which have recently undergone a collision, or perhaps a single star which has been partially tidally disrupted (Manukian et al. 2013). While we favor a stellar model, this question will be soon addressed, as G2 should remain intact through its periapse if this is indeed correct.

The authors acknowledge the invaluable feedback from the referee and conference attendees at workshops where this work has been presented (AAS 221st Meeting, Keck 20th Anniversary Celebration, Galactic Nuclei Ringberg Workshop). Support for this work was provided by NSF grant AST-0909218. Data presented herein were taken at the Keck Observatory, which is operated as a scientific partnership among Caltech, UC, and NASA; the Observatory was made possible by the generous financial support of the Keck Foundation.

Facilities: Keck:II (LGS AO, NIRC2, OSIRIS), Keck:I (LGS AO, OSIRIS)

\section{REFERENCES}

Alexander, T., \& Livio, M. 2004, ApJL, 606, L21

Anninos, P., Fragile, P. C., Wilson, J., \& Murray, S. D. 2012, ApJ, 759,132

Bartko, H., Martins, F., Fritz, T. K., et al. 2009, ApJ, 697, 1741

Bartos, I., Haiman, Z., Kocsis, B., \& Marka, S. 2013, PhRvL, 110, 221102

Burkert, A., Schartmann, M., Alig, C., et al. 2012, ApJ, 750, 58

Chin, J. C. Y., Wizinowich, P., Campbell, R., et al. 2012, Proc. SPIE, 8447, 84474F

Cuadra, J., Nayakshin, S., Springel, V., \& Di Matteo, T. 2006, MNRAS, 366,358

Diolaiti, E., Bendinelli, O., Bonaccini, D., et al. 2000, A\&AS, 147, 335

Do, T., Ghez, A. M., Morris, M. R., et al. 2009, ApJ, 703, 1323

Eckart, A., Mužić, K., Yazici, S., et al. 2013, A\&A, 551, A18

Feroz, F., \& Hobson, M. P. 2008, MNRAS, 384, 449

Genzel, R., Pichon, C., Eckart, A., Gerhard, O. E., \& Ott, T. 2000, MNRAS, 317,348
Ghez, A. M., Hornstein, S. D., Lu, J. R., et al. 2005a, ApJ, 635, 1087 Ghez, A. M., Salim, S., Hornstein, S. D., et al. 2005b, ApJ, 620, 744 Ghez, A. M., Salim, S., Weinberg, N. N., et al. 2008, ApJ, 689, 1044

Gillessen, S., Genzel, R., Fritz, T. K., et al. 2012, Natur, 481, 51

Gillessen, S., Genzel, R., Fritz, T. K., et al. 2013, ApJ, 763, 78

Hartkopf, W. I., Mason, B. D., \& Worley, C. E. 2001, AJ, 122, 3472

Hornstein, S. D., Matthews, K., Ghez, A. M., et al. 2007, ApJ, 667, 900

Kassim, N. E., Hyman, S. D., Intema, H., Clarke, T. E., \& Subrahmanyan, R. 2013, ATel, 4922, 1

Larkin, J., Barczys, M., Krabbe, A., et al. 2006, NewAR, 50, 362

Levin, Y., \& Beloborodov, A. M. 2003, ApJL, 590, L33

Lu, J. R., Ghez, A. M., Hornstein, S. D., et al. 2009, ApJ, 690, 1463

Lucy, L. B. 1974, AJ, 79, 745

Lucy, L. B., \& Hook, R. N. 1992, in ASP Conf. Ser. 25, Astronomical Data Analysis Software and Systems I, ed. D. M. Worrall, C. Biemesderfer, \& J. Barnes (San Francisco, CA: ASP), 277

Manukian, H., Guillochon, J., Ramirez-Ruiz, E., \& O'Leary, R. M. 2013, ApJL, 771, L28

Meyer, L., Ghez, A. M., Schödel, R., et al. 2012, Sci, 338, 84

Miralda-Escudé, J. 2012, ApJ, 756, 86

Morris, M. 2012, Natur, 481, 32

Mościbrodzka, M., Shiokawa, H., Gammie, C. F., \& Dolence, J. C. 2012, ApJL, 752, L1

Murray-Clay, R. A., \& Loeb, A. 2012, NatCo, 3E1049M

Narayan, R., Özel, F., \& Sironi, L. 2012, ApJL, 757, L20

Ozernoy, L. M., Genzel, R., \& Usov, V. V. 1997, MNRAS, 288, 237

Paumard, T., Genzel, R., Martins, F., et al. 2006, ApJ, 643, 1011

Richardson, W. H. 1972, JOSA, 62, 55

Sadowski, A., Narayan, R., Sironi, L., \& Ozel, F. 2013a, MNRAS, 433,2165

Sadowski, A., Sironi, L., Abarca, D., et al. 2013b, MNRAS, 432, 478

Saitoh, T. R., Makino, J., Asaki, Y., et al. 2012, arXiv:1212.0349

Schartmann, M., Burkert, A., Alig, C., et al. 2012, ApJ, 755, 155

Schödel, R., Najarro, F., Muzic, K., \& Eckart, A. 2010, A\&A, 511, A18

Scoville, N., \& Burkert, A. 2013, ApJ, 768, 108

van Dam, M. A., Bouchez, A. H., Le Mignant, D., et al. 2006, PASP, 118,310

Witzel, G., Eckart, A., Bremer, M., et al. 2012, ApJS, 203, 18

Wizinowich, P. L., Le Mignant, D., Bouchez, A. H., et al. 2006, PASP, 118, 297

Yelda, S., Ghez, A. M., Lu, J. R., et al. 2013, ApJ, submitted

Yelda, S., Lu, J. R., Ghez, A. M., et al. 2010, ApJ, 725, 331

Yusef-Zadeh, F., \& Wardle, M. 2013, ApJL, 770, L21 